\title{
Multimodality Intravascular Imaging Assessment of Plaque Erosion versus Plaque Rupture in Patients with Acute Coronary Syndrome
}

Jee Eun Kwon, MD1, Wang Soo Lee, MD1, Gary S. Mintz, MD², Young Joon Hong, MD³, Sung Yun Lee, MD4 Ki Seok Kim, MD², Joo-Yong Hahn, MD ${ }^{6}$, Kaup Sharath Kumar, MD¹, Hoyoun Won, MD¹, Seong Hyeop Hyeon, MD', Seung Yong Shin, MD', Kwang Je Lee, MD1', Tae Ho Kim, MD¹, Chee Jeong Kim, MD1', and Sang Wook Kim, MD¹

${ }^{1}$ Heart Research Institute, Cardiovascular-Arrhythmia Center, College of Medicine, Chung-Ang University Hospital, Seoul, Korea, ${ }^{2}$ Cardiovascular Research Foundation, New York, USA, ${ }^{3}$ Chonnam National University Hospital, Gwangju, ${ }^{4}$ Inje University Ilsan Paik Hospital, Koyang, ${ }^{5}$ Jeju National University Hospital, Jeju, ${ }^{6}$ Samsung Medical Center, Seoul, Korea

Background and Objectives: We assessed plaque erosion of culprit lesions in patients with acute coronary syndrome in real world practice. Subjects and Methods: Culprit lesion plaque rupture or plaque erosion was diagnosed with optical coherence tomography (OCT). Intravascular ultrasound (IVUS) was used to determine arterial remodeling. Positive remodeling was defined as a remodeling index (lesion/ reference EEM [external elastic membrane area) >1.05.

Results: A total of 90 patients who had plaque rupture showing fibrous-cap discontinuity and ruptured cavity were enrolled. 36 patients showed definite OCT-plaque erosion, while 7 patients had probable OCT-plaque erosion. Overall, 26\% (11/43) of definite/probable plaque erosion had non-ST elevation myocardial infarction (NSTEMI) while 35\% (15/43) had ST elevation myocardial infarction (STEMI). Conversely, $14.5 \%$ (13/90) of plaque rupture had NSTEMI while 71\% (64/90) had STEMI $(p<0.0001)$. Among plaque erosion, white thrombus was seen in 55.8\% (24/43) of patients and red thrombus in 27.9\% (12/43) of patients. Compared to plaque erosion, plaque rupture more often showed positive remodeling $(\mathrm{p}=0.003)$ with a larger necrotic core area examined by virtual histology $(\mathrm{VH})-\mathrm{IVUS}$, while negative remodeling was prominent in plaque erosion. Overall, 65\% 28/43 of plaque erosions were located in the proximal $30 \mathrm{~mm}$ of a culprit vessel-similar to plaque ruptures (72\%, 65/90, $p=0.29)$.

Conclusion: Although most of plaque erosions show nearly normal coronary angiogram, modest plaque burden with negative remodeling and an uncommon fibroatheroma might be the nature of plaque erosion. Multimodality intravascular imaging with OCT and VH-IVUS showed fundamentally different pathoanatomic substrates underlying plaque rupture and erosion. (Korean Circ J 2016;46(4):499-506)

KEY WORDS: Optical coherence tomography; Atherosclerotic plaque; Acute coronary syndrome.

Received: August 11, 2015

Revision Received: November 12, 2015

Accepted: December 24, 2015

Correspondence: Wang Soo Lee, MD, Heart Research Institute, Cardiovascular-Arrhythmia Center, College of Medicine, Chung-Ang University Hospital, 102 Heukseok-ro, Dongjak-gu, Seoul 06973, Korea

Tel: 82-2-6299-2871, Fax: 82-2-823-0160

E-mail:wslee1227@cau.ac.kr

- The authors have no financial conflicts of interest.

This is an Open Access article distributed under the terms of the Creative Commons Attribution Non-Commercial License (http://creativecommons. org/licenses/by-nc/3.0) which permits unrestricted non-commercial use, distribution, and reproduction in any medium, provided the original work is properly cited.

\section{Introduction}

Pathologic studies ${ }^{1-3)}$ have shown plaque ruptures in 55-70\% of patients and plaque erosion in $25-40 \%$ of patients with sudden coronary death. However, the mechanisms of plaque erosion remain unclear and the clinical importance and impact have been underestimated due to limited resolution of intravascular ultrasound (IVUS) and computed tomography. ${ }^{4}$ In addition, small plaque ruptures and unruptured thin-capped fibroatheromas (TCFA) with superimposed thrombosis have not been fully evaluated. ${ }^{516)}$

Optical coherence tomography (OCT) provides high resolution cross-sectional imaging of atheromatous plaques in vivo. Previous studies have shown that OCT can detect TCFAs, plaque ruptures, 
plaque erosions, and intracoronary thrombus with greater accuracy than IVUS, although the limited penetration of OCT especially in the setting of high-risk plaques with superimposed red thrombus can affect the quantitative assessment of lipid and remodeling. ${ }^{7-13)}$ We hypothesized that combined imaging with OCT and radiofrequency (virtual histology [VH])-IVUS would overcome the limitation of each imaging modality. Therefore, we conducted a multicenter study using both OCT and VH-IVUS to assess culprit lesion morphology of patients presenting acute coronary syndromes (ACS).

\section{Subjects and Methods}

\section{Study population}

A total of 133 consecutive patients underwent percutaneous coronary intervention with both OCT and VH-IVUS imaging to assess the culprit lesion at five centers participating in Korea Cardiovascular Imaging OCT registry. The study protocol was approved by the Institutional Review Board of each institution. Written informed consent was obtained from all patients.

Patients with bifurcation lesions, ostial lesions, vein graft lesions, multiple culprit lesions, arteries with previous stent placement, debulking or plaque modification procedures before intravascular imaging, prominent red thrombus, end-stage renal disease, and severe left ventricular dysfunction were excluded. Thrombus aspiration was performed prior to OCT and IVUS imaging using an aspiration catheter (Thrombuster ${ }^{\circledR}$, Kaneka Co., Osaka, Japan) according to operator discretion, but typically for large thrombi in the setting of ST-segment elevation myocardial infarction (STEMI) because such thrombi would affect the penetration of OCT and the accuracy of VH-IVUS.

STEMI was diagnosed based on continuous chest pain for at least $30 \mathrm{~min}$, arrival at the hospital within 6 hour from the onset of symptoms, ST-segment elevation $>0.1 \mathrm{mV}$ in two or more contiguous leads or newly found left bundle-branch block on the 12-lead electrocardiogram (ECG), and elevated cardiac markers (plasma creatine kinase-myocardial band or troponin I). Non-STsegment elevation myocardial infarction (NSTEMI) was defined as ischemic symptoms with elevated cardiac markers in the absence of ST-segment elevation on ECG. Unstable angina pectoris was defined as new onset/accelerating chest symptoms on exertion or rest angina within two weeks. Culprit lesion was identified based on coronary angiogram, stress test, ECG, and/or echocardiogram.

Hypertension was defined as systolic blood pressure $\geq 140$ $\mathrm{mmHg}$ or diastolic blood pressure $\geq 90 \mathrm{mmHg}$ or current use of antihypertensive treatment. Diabetes mellitus was defined as hemoglobin $A_{1} C \geq 6.5 \mathrm{mg} / \mathrm{dL}$ or taking medication for diabetes mellitus. Dyslipidemia was classified as total cholesterol level $\geq 220$ $\mathrm{mg} / \mathrm{dL}$, low-density lipoprotein cholesterol level $\geq 140 \mathrm{mg} / \mathrm{dL}$, high-density lipoprotein cholesterol level $<40 \mathrm{mg} / \mathrm{dL}$, or taking medication for dyslipidemia.

\section{Angiographic analysis}

Coronary angiography was performed after intracoronary administration of $200 \mu \mathrm{g}$ of nitroglycerin. All angiograms were analyzed with an automated edge-detection algorithm (AI 1000, GE Medical Systems, Milwaukee, WI, USA) using standard protocols. Minimal luminal diameter, \% diameter stenosis, and reference vessel diameter were measured before coronary intervention. Lesion measurements were performed in the "worst" view, and the end-diastolic frame was selected for analysis.

\section{Optical coherence tomography imaging and analysis}

A commercially available frequency domain OCT system (C7XR or Ilumien System, Light Lab Imaging, Inc., St. Jude Medical, Westford, MA, USA) and a 0.014-inch wire-type imaging catheter (ImageWire, St. Jude Medical, Westford, MA, USA) were used. Motorized ImageWire pull-back at $10 \mathrm{~mm} / \mathrm{s}$ was performed during simultaneous injection of a viscous iso-osmolar contrast solution.

OCT plaque composition was analyzed as described previously. ${ }^{14)}$ OCT-identified TCAF was defined as a fibrous cap thickness $\leq 65$ $\mu \mathrm{m}$ at the thinnest part with an angle of the lipid $\geq 180^{\circ}$. Definite OCT-plaque erosion was defined as the presence of intracoronary thrombus attaching to the luminal surface without detectable signs of fibrous cap rupture. Probable OCT-plaque erosion was defined as luminal surface irregularity at the culprit lesion in the absence of thrombus. Plaque rupture was identified by the presence of fibrouscap discontinuity and cavity formation in the plaque. All OCT images were analyzed using certified Offline Review Workstation (St. Jude Medical, Westford, MA, USA) at an independent core laboratory (Heart Research Institute, Seoul, Korea) by two analysts (DWK and YMJ) who were blinded to patient information.

\section{Intravascular ultrasound examination and analysis}

A commercially available VH-IVUS system (Volcano Therapeutics, Rancho Cordova, CA, USA) and $20 \mathrm{MHz}$ transducers were used for all IVUS examinations. Greyscale IVUS analysis was performed according to criteria established by the American College of Cardiology clinical expert consensus document on IVUS'15) using planimetry software (Echoplaque, INDEC Systems Inc., Mountain View, CA, USA). IVUS imaging was performed after intracoronary administration of $200 \mu \mathrm{g}$ of nitroglycerin. The IVUS catheter was advanced $10 \mathrm{~mm}$ distal to the target lesion, and imaging was 
performed retrograde back to the aorto-ostial junction using an ECG-gated automatic pullback device. Studies were recorded onto electronic media for off-line analysis. VH-IVUS plaque composition and lesion phenotype were assessed after defining the two standard VH-IVUS regions-of-interest: inner border (lumen, excluding IVUSdetectable thrombus) and outer border (external elastic membrane). VH-IVUS pathologic intimal thickening (PIT), thin cap fibroatheroma (TCFA), thick-capped fibroatheroma (ThCFA), and fibrotic or fibrocalcific plaque were defined as published. ${ }^{16)}$ TCFA was $>10 \%$ confluent necrotic core (NC) with $>30^{\circ} \mathrm{NC}$ abutting the lumen in $\geq 3$ consecutive frames without evidence of fibrous cap. ThCFA was a fibroatheroma ( $>10 \%$ of confluent NC in $\geq 3$ consecutive frames) with a definable fibrous cap. ${ }^{16)}$ Positive remodeling was defined as a remodeling index (lesion/reference external elastic membrane [EEM] area) $>1.05$. Intermediate remodeling was defined as a remodeling index between 0.95 and 1.05 , and negative remodeling as a remodeling index $<0.95$. Culprit lesions on VH-IVUS and OCT studies were compared using reproducible axial landmarks (usually the aorto-ostial junction, a large proximal side branch, and/or reference segment calcific deposits) and know pullback speeds.

\section{Statistical analysis}

Results are expressed as mean ${ }_{ \pm} S D$ (standard deviation). Frequencies are expressed in numbers and relative percentages.

Table 1. Baseline characteristics

\begin{tabular}{|c|c|c|c|}
\hline & Plaque rupture $(n=90)$ & Plaque erosion $(n=43)$ & p \\
\hline Age (years) & $60.11 \pm 12.51$ & $61.98 \pm 9.99$ & 0.3938 \\
\hline Female gender & $17(18.9)$ & 7 (16.3) & 0.7143 \\
\hline Diabetes mellitus & $20(22.2)$ & $12(27.9)$ & 0.4731 \\
\hline Hypertension & $37(41.1)$ & $21(48.8)$ & 0.4007 \\
\hline Current smoker & $39(43.3)$ & $15(34.9)$ & 0.6458 \\
\hline Hyperlipidemia & $9(10.1)$ & $4(9.3)$ & 0.8836 \\
\hline Total cholesterol (mg/dL) & $187.3 \pm 41.5$ & $179.7 \pm 47.49$ & 0.3521 \\
\hline Clinical presentation & & & $<0.0001$ \\
\hline Unstable angina & $13(14.5)$ & $17(39)$ & \\
\hline NSTEMI & $13(14.5)$ & $11(26)$ & \\
\hline STEMI & $64(71)$ & $15(35)$ & \\
\hline Diseased vessels & & & 0.5322 \\
\hline 1 vessel & $33(36.7)$ & $11(34.4)$ & \\
\hline 2 vessel & $33(36.7)$ & $15(46.9)$ & \\
\hline 3 vessel & $24(26.7)$ & $6(18.8)$ & \\
\hline Target lesion & & & 0.4281 \\
\hline$L A D$ & $47(52.2)$ & $27(64.3)$ & \\
\hline LCX & $9(10.0)$ & $3(7.14)$ & \\
\hline RCA & $34(37.9)$ & $12(28.6)$ & \\
\hline \multicolumn{4}{|l|}{ Quantitative coronary angiography } \\
\hline Lesion length (mm) & $17.98 \pm 7.03$ & $2.26 \pm 1.15$ & $<0.0001$ \\
\hline Diameter stenosis & $81.0 \pm 19.3$ & $17.5 \pm 14.2$ & $<0.0001$ \\
\hline Reference vessel diameter (mm) & $3.47 \pm 0.61$ & $2.98 \pm 0.97$ & 0.0047 \\
\hline TIMI grade & & & $<0.0001$ \\
\hline 0 & $44(48.9)$ & $4(9.3)$ & \\
\hline 1 & $2(2.2)$ & $0(0.0)$ & \\
\hline 2 & 23 (25.6) & $6(13.9)$ & \\
\hline 3 & 21 (23.3) & 33 (76.7) & \\
\hline
\end{tabular}

Values are presented as means \pm standard deviation or $n(\%)$. NSTEMI: non-ST elevation myocardial infarction, STEMI: ST elevation myocardial infarction, LAD: left anterior descending artery, LCX: left circumflex artery, RCA: right coronary artery, TIMI: thrombolysis in myocardial infarction 
Distributions of continuous variables were evaluated. For continuous variables, student $t$ test was performed to compare the difference between measurements, and categorical variables were compared using chi-square test. All analyses were performed using standard statistical software (SPSS version 18.0, IBM, Chicago, IL, USA). All $p$ values were two-tailed, and statistical significance was considered when $p$ value was less than 0.05 .

\section{Results}

\section{Clinical and angiographic findings}

A total of 133 consecutive patients had OCT and VH-IVUS studies that were amenable to analysis, including 90 patients who had OCT-plaque rupture and 43 patients who had OCT-plaque erosion (36 definite and 7 probable). The baseline characteristics of the study population are shown in Table 1. Patient age, gender, and clinical risk factors in the plaque rupture group were similar to those in the plaque erosion group. However, the prevalence of plaque rupture was significantly higher in STEMI, 71\% (64/90) $(p<0.0001)$ compared to the prevalence of plaque erosion, while the prevalence of plaque erosion was significantly higher in unstable angina/NSTEMI $(68 \%$ [28/43]) $(p<0.0001)$ compared to that of plaque rupture.

Quantitative angiographic analysis showed that plaque erosion lesions were significantly shorter than plaque ruptures $(p<0.0001)$, had significantly smaller reference vessel diameter $(p=0.0047)$, less severe diameter stenosis $(p<0.0001)$, and higher rate of Thrombolysis in Myocardial Infarction (TIMI) grade 3. $(p<0.0001)$.

Table 2. IVUS comparison of plaque rupture sites versus plaque erosion sites

\begin{tabular}{|c|c|c|c|}
\hline & Plaque rupture $(n=90)$ & Plaque erosion $(n=43)$ & $\mathbf{p}$ \\
\hline \multicolumn{4}{|l|}{ Proximal reference segment } \\
\hline EEM area $\left(\mathrm{mm}^{2}\right)$ & $18.66 \pm 5.33$ & $16.43 \pm 4.95$ & 0.0286 \\
\hline Lumen area $\left(\mathrm{mm}^{2}\right)$ & $9.36 \pm 2.91$ & $8.77 \pm 3.28$ & 0.3100 \\
\hline Plaque area $\left(\mathrm{mm}^{2}\right)$ & $10.09 \pm 6.66$ & $7.84 \pm 3.06$ & 0.0466 \\
\hline \multicolumn{4}{|l|}{ Rupture vs. erosion sites } \\
\hline $\operatorname{EEM}\left(\mathrm{mm}^{2}\right)$ & $17.68 \pm 5.93$ & $15.07 \pm 7.48$ & 0.0359 \\
\hline Lumen area $\left(\mathrm{mm}^{2}\right)$ & $6.08 \pm 2.85$ & $7.02 \pm 3.29$ & 0.1100 \\
\hline Plaque area $\left(\mathrm{mm}^{2}\right)$ & $11.61 \pm 4.60$ & $7.29 \pm 3.38$ & $<0.0001$ \\
\hline Plaque burden (\%) & $65.1 \pm 12.15$ & $50.50 \pm 16.63$ & $<0.0001$ \\
\hline Fibrotic area $\left(\mathrm{mm}^{2)}\right.$ & $4.57 \pm 2.39$ & $2.42 \pm 1.9$ & $<0.0001$ \\
\hline Fibrofatty area $\left(\mathrm{mm}^{2}\right)$ & $1.13 \pm 1.42$ & $0.72 \pm 0.94$ & 0.0576 \\
\hline Necrotic core area $\left(\mathrm{mm}^{2}\right)$ & $2.11 \pm 1.38$ & $0.90 \pm 1.00$ & $<0.0001$ \\
\hline Dense calcium area $\left(\mathrm{mm}^{2}\right)$ & $0.67 \pm 0.76$ & $0.36 \pm 0.62$ & 0.0287 \\
\hline \% Fibrotic area & $55.03 \pm 13.65$ & $71.73 \pm 105.9$ & 0.1400 \\
\hline$\%$ Fibrofatty area & $11.98 \pm 11.76$ & $15.23 \pm 16.51$ & 0.2700 \\
\hline \% Necrotic core area & $24.74 \pm 11.07$ & $15.06 \pm 12.47$ & $<0.0001$ \\
\hline \% Dense calcium area & $8.24 \pm 8.96$ & $6.34 \pm 10.18$ & 0.2900 \\
\hline Remodeling Index & $1.12 \pm 0.29$ & $0.94 \pm 0.36$ & 0.0050 \\
\hline Remodeling & & & 0.0030 \\
\hline Positive & $52(57.7)$ & $14(32.5)$ & \\
\hline Intermediate & $15(16.6)$ & $4(9.3)$ & \\
\hline Negative & $23(25.5)$ & $25(58.1)$ & \\
\hline \multicolumn{4}{|l|}{ Distal reference segment } \\
\hline EEM area $\left(\mathrm{mm}^{2}\right)$ & $13.75 \pm 5.75$ & $12.83 \pm 4.63$ & 0.3743 \\
\hline Lumen area $\left(\mathrm{mm}^{2}\right)$ & $7.54 \pm 3.20$ & $7.17 \pm 2.82$ & 0.5332 \\
\hline Plaque area $\left(\mathrm{mm}^{2}\right)$ & $6.74 \pm 4.18$ & $5.67 \pm 2.85$ & 0.0961 \\
\hline
\end{tabular}

Values are presented as means \pm standard deviation or $n(\%)$. IVUS: intravascular ultrasound, EEM: external elastic membrane area 


\section{Intravascular ultrasound analysis of plaque ruptures and plaque erosions}

At the site of plaque erosion or plaque rupture, EEM area was larger in plaque ruptures $(p=0.0359)$ with larger plaque burden $(p<0.0001)$, more positive remodeling $(p=0.029)$ and larger $N C$ $(p<0.0001)$. Conversely, 58.1\% (25 of 43) definite/probable plaque erosions showed negative remodeling. Based on VH-IVUS, 83\% (36 of 43) of plaque erosions were fibrotic, and 17\% (7 of 43) were fibrocalcific. Compared to plaque erosions, 43.3\% (39 of 90) of plaque ruptures had the appearance of VH-TCFA, 12.2\% (11 of 90) were PIT, 17.7\% (16 of 90) were fibrotic, 20\% (18 of 90) were fibrocalcific, and 6.6\% (6 of 90) were thick cap fibroatheromas $(p<0.0001$, Table 2).

\section{Optical coherence tomography analysis of plaque ruptures and erosions}

The OCT findings of plaque erosion are shown in Fig. 1 and Table 3. Based on OCT, 60\% (26 of 43) of definite/probable plaque erosions were fibrotic, 16\% (7 of 43) were fibrocalcific, 11.6\% (5 of 43) were lipidic, and $11.6 \%$ (5 of 43 ) had thick capped fibroatheroma. None of the plaque erosion had the appearance of OCT-TCFA, and three of $43(7 \%)$ definite/probable plaque erosions contained calcific nodule. Conversely, 52\% (47 of 90) of ruptured plaques had the appearance of OCT-TCFA with a fibrous cap thickness of $51 \pm 7.05$ $\mu \mathrm{m}$. Approximately $4.4 \%$ (4 of 90 ) of ruptured plaques were fibrotic, $28.8 \%$ (26 of 90) were lipidic, 13.3\% (12 of 90) were fibrocalcific, and $1.1 \%$ (1 of 90) had the appearance of a thick cap fibroatheroma ( $p<0.0001$ vs. plaque erosions).

Intracoronary thrombus was found in $83.7 \%$ (36/43) of plaque erosion; white thrombus in 55.8\% (24/43) with red thrombus in $27.9 \%$ (12/43), and no detectable thrombus in 16.3\% (7/43) of plaque erosion. Conversely, intracoronary thrombus was observed in all cases of plaque rupture which was mostly red thrombus (92\% [83/90]) ( $p<0.0001$ vs. plaque erosions).

Overall, 46.5\% (20/43) of plaque erosions were located proximal to the minimal lumen area (MLA) site while 27.9\% (12/43) were distal to the MLA site and 25.6\% (11/43) were at the MLA site. Conversely, 57.7\% (52/90) of plaque ruptures were located proximal to MLA site, 42.3\% (38/90) were distal to the MLA site, and none was located at the MLA site ( $p=0.002$ vs. plaque erosions).

The distance from the coronary ostium to the site of plaque
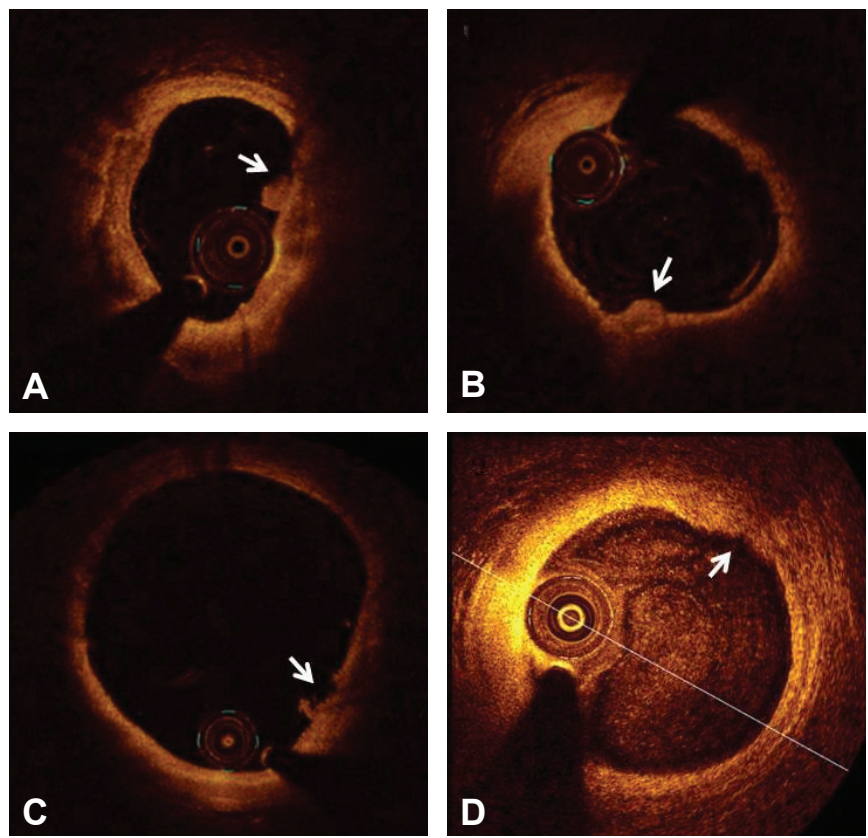

Fig. 1. Optical coherence tomography finding of plaque erosion. (A-C) Definite plaque erosion, intracoronary thrombi (arrow) attaching to the luminal surface were noted without detectable signs of fibrous cap rupture. (D) Probable plaque erosion, coronary luminal surface irregularity (arrow) with the absence of thrombus was noted at the culprit lesion.

Table 3. Comparative findings of OCT in plaque rupture and plaque erosion

\begin{tabular}{lcc}
\hline \multicolumn{1}{|c}{} & Plaque rupture $(\mathbf{n = 9 0 )}$ & Plaque erosion $(\mathbf{n = 4 3 )}$ \\
\hline Plaque component & & $\mathbf{p}$ \\
\hline Fibrotic & $4(4.4)$ & $26(60)$ \\
\hline Lipidic & $26(28.8)$ & $5(11.6)$ \\
\hline Fibrocalcific & $12(13.3)$ & $7(16)$ \\
\hline Thick cap fibroatheroma & $1(1.1)$ & $5(11.6)$ \\
\hline Thin-capped fibroatheroma & $47(52)$ & 0 \\
\hline Thrombus & & $24(55.8)$ \\
\hline White thrombus & $7(8)$ & $12(27.9)$ \\
\hline Red thrombus & $83(92)$ & $7(16.3)$ \\
\hline No thrombus & 0 & $<0.0001$ \\
\hline
\end{tabular}

Values are presented as n (\%). OCT: optical coherence tomography 


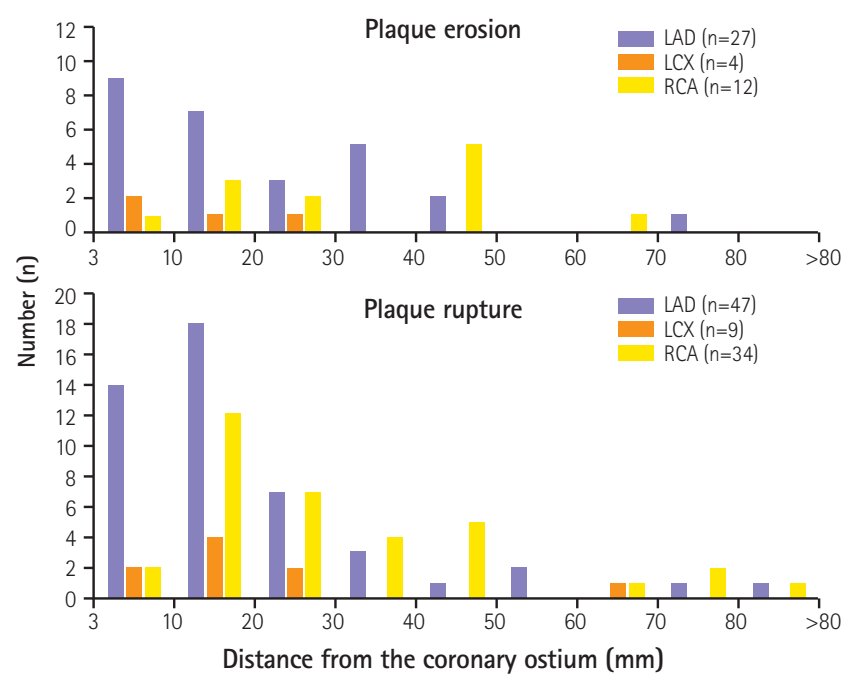

Fig. 2. Frequency distribution of plaque rupture/erosion at culprit according to distance from each coronary ostium of the $L A D$, the $L C X$, and the RCA. Plaque rupture and plaque erosions are shown separately. The distribution pattern of plaque rupture was similar $(p=0.29)$ to that of plaque erosions. LAD: left anterior descending coronary artery, LCX: left circumflex coronary artery, RCA: right coronary artery.

rupture was $23.54 \pm 18.42 \mathrm{~mm}$, similar to the distance from the coronary artery ostium to the site of plaque erosion $(23.37 \pm 17.82$ $\mathrm{mm}, \mathrm{p}=0.96)$. Overall, $65 \%$ (28/43) of plaque erosions were located in the proximal $30 \mathrm{~mm}$ of a culprit vessel, similar to plaque ruptures (72\%, 65 of 90, p=0.29) (Fig. 2), although erosions in right coronary artery (RCA) appeared to be more diffusely distributed compared to erosions in left anterior descending coronary artery or left circumflex coronary artery and also compared to ruptures in RCA.

\section{Discussion}

The main findings of the present study were as follows: (1) Plaque rupture was often associated with positive remodeling, large plaque burden, and red thrombus. (2) Plaque erosion was associated with negative remodeling, a modest plaque burden, white thrombus, uncommon features of a fibroatheroma, and proximal distribution.

Plaque rupture has been reported to be the main cause of acute coronary syndrome (ACS) with plaque erosion responsible for most of other cases. ${ }^{1-3)}$ Necrotic cores were uncommon in erosion lesions and a thick fibrous cap separated the lumen from the necrotic core when it exists. Erosions occurred on top of lesions which were rich in smooth muscle cells and proteoglycans. ${ }^{2}$ Plaque erosion is prevalent in female patients less than 50 years old ${ }^{11}$ and is associated with smoking. ${ }^{3)}$ In this current study, patients with plaque erosions vs plaque ruptures had similar age and gender profiles. However, unstable angina and NSTEMI were found to be more frequent in plaque erosions while plaque rupture seen in 71\% of patients with STEMI. These findings were consistent with results of previous studies that plaque erosions are infrequent in STEMI patients on admission. ${ }^{211117)}$

The low resolution of IVUS precludes the evaluation of erosions. However, a limited number of imaging studies have used OCT to evaluate the role of plaque erosion in the pathophysiology of ACS in vivo. OCT was superior to coronary angioscopy and IVUS in the detection of fibrous cap erosion, and fibrotic plaque was most frequent in plaque erosion. However, coronary artery remodeling was not defined due to limited number and heterogenous clinical entities. $^{9-11)}$

Ruptured plaques of culprit lesion were predominately located in the proximal segments of the coronary arteries. The proximal $30 \mathrm{~mm}$ was associated with plaque rupture, TCFA, and lower minimal cap thickness. ${ }^{18-21)}$ In this study, the longitudinal distribution of plaque erosions was identical to plaque rupture mostly located in proximal portion of the coronary artery except the RCA with less plaque area and negative remodeling. Positive remodeling is typically considered to be indicative of an unstable lesion, while negative remodeling is indicative of stable coronary artery disease. ${ }^{22)}$ However, the current study suggests that negative remodeling could be a feature of plaque erosions and therefore, it is not always benign, consistent with recent data from Providing Regional Observations to Study Predictors of Events in the Coronary Tree (PROSPECT) study. ${ }^{23)}$

More than half of plaque erosions in the current study appeared to be fibrotic plaque rather than TCFA. Plaque erosions is frequently found with white thrombus compared to plaque rupture in patients with ACS associated with red thrombus. ${ }^{11)}$ Autopsy studies ${ }^{24)}$ have reported that more than $88 \%$ of coronary thrombi overlying plaque erosions showed later stages of healing that are characterized by invasion of organized layers of smooth muscle cells and endothelial cells with various degrees of platelet/fibrin layering. However, only $50 \%$ of thrombi showed evidence of healing in patients with plaque rupture. ${ }^{24)}$ Fibrin rich red thrombus was frequently found over ruptured plaque, whereas platelet rich white thrombus was the predominant type of thrombus formed over plaque erosion and calcified nodule. Plaque rupture induces massive thrombus formation at the culprit site. In contrast, plaque erosion seems to result in less thrombus burden, preserved vascular structure, and larger lumen.124) In current study, 93\% of plaque erosions showed $<50 \%$ diameter stenosis. More importantly, it might provoke a misdiagnosis in ACS patient who showed near normal or normal coronary angiogram.

This study has several limitations. First, it was a observational study with a relatively small sample size. The accuracy of OCT and 
VH-IVUS analysis was reduced in the setting of intracoronary thrombus. Although patients with culprit lesion with prominent thrombi burden were excluded, the presence of thrombus overlying the culprit lesion might reduce the ability to assess underlying plaque characteristics by OCT. In addition, we excluded ambiguous plaque ruptures/erosion with prominent thrombus. Thrombectomy itself can affect plaque morphology and the accuracy of data. Plaque erosion and calcified nodule as detected by OCT were not validated by autopsy study. Patients with large MI and heart failure patients were less likely to undergo pre-intervention OCT imaging. In addition, we could not assess the pathophysiologic mechanism in this study. However, further study should identify the pathophysiologic mechanism that causes plaque erosion. Although the complication rate was very low, we could not exclude the possibility that plaque erosion might be developed during contrast injection for OCT.

\section{Conclusion}

Although plaque erosion shows a near normal coronary angiogram, a modest plaque burden with negative remodeling and uncommon fibroatheroma might be the nature of plaque erosion, which should not be misdiagnosed. Multimodality intravascular imaging with OCT and VH-IVUS showed fundamentally different pathoanatomic substrates underlying plaque rupture and erosion, indicating that they are distinct lesion phenotypes.

\section{Acknowledgments}

The authors thank Dal Won Kang and Young Mi Jo independent researchers of the core lab for their efforts to analyze OCT and IVUS, and Kyung Sook Park (MPH) for her statistical assistance.

\section{References}

1. Farb A, Burke AP, Tang AL, et al. Coronary plaque erosion without rupture into a lipid core: a frequent cause of coronary thrombosis in sudden coronary death. Circulation 1996;93:1354-63.

2. Virmani $R, B$ urke $A P$, Farb $A$, Kolodgie FD. Pathology of the vulnerable plaque. J Am Coll Cardio/ 2006;47(8 Suppl):C13-8.

3. Burke AP, Farb A, Malcom GT, Liang Y, Smialek J, Virmani R. Effect of risk factors on the mechanism of acute thrombosis and sudden coronary death in women. Circulation 1998;97:2110-6.

4. Hong YJ, Ahn Y, Jeong MH. Role of Intravascular Ultrasound in Patients with Acute Myocardial Infarction. Korean Circ J 2015:45:259-65.
5. Sanidas EA, Maehara A, Mintz GS, et al. Angioscopic and virtual histology intravascular ultrasound characteristics of culprit lesion morphology underlying coronary artery thrombosis. Am J Cardiol 2011;107:1285-90.

6. Kim SW, Hong YJ, Mintz GS, et al. Relation of ruptured plaque culprit lesion phenotype and outcomes in patients with ST elevation acute myocardial infarction. Am J Cardiol 2012;109:794-9.

7. Yabushita $H_{1}$ Bouma BE, Houser SL, et al. Characterization of human atherosclerosis by optical coherence tomography. Circulation 2002;106:1640-5.

8. Jang IK, Tearney GJ, MacNeill B, et al. In vivo characterization of coronary atheroscleroticplaque by use of optical coherence tomography. Circulation 2005;111:1551-5.

9. Kubo T, Imanishi T, Takarada S, et al. Assessment of culprit lesion morphology in acute myocardial infarction: ability of optical coherence tomography compared with intravascular ultrasound and coronary angioscopy. J Am Coll Cardio/ 2007;50:933-9.

10. Rathore S, Terashima M, Matsuo H, et al. Association of coronary plaque composition and arterial remodeling: an optical coherence tomography study. Atherosclerosis 2012;221:405-15.

11. Jia $H$, Abtahian $F$, Aguirre $A D$, et al. In vivo diagnosis of plaque erosion and calcified nodule in patients with acute coronary syndrome by intravascular optical coherence tomography. J Am Coll Cardio/ 2013;62:1748-58.

12. Ino $Y$, Kubo T, Tanaka A, et al. Difference of culprit lesion morphologies between ST-segment elevation myocardial infarction and non-ST-segment elevation acute coronary syndrome: an optical coherence tomography study. JACC Cardiovasc Interv 2011;4:76-82.

13. Cho YK, Hur SH. Practical Application of Coronary Imaging Devices in Cardiovascular Intervention, Korean Circ J 2015;45:87-95.

14. Tearney GJ, Regar E, Akasaka T, et al; International Working Group for Intravascular Optical Coherence Tomography (IWG-IVOCT). Consensus standards for acquisition, measurement, and reporting of intravascular optical coherence tomography studies: a report from the International Working Group for Intravascular Optical Coherence Tomography Standardization and Validation. J Am Coll Cardiol 2012;59:1058-72.

15. Mintz GS, Nissen SE, Anderson WD, et al. American College of Cardiology Clinical Expert Consensus Document on Standards for Acquisition, Measurement and Reporting of Intravascular Ultrasound Studies (IVUS). A report of the American College of Cardiology Task Force on Clinical Expert Consensus Documents. J Am Coll Cardiol 2001;37:1478-92.

16. García-García HM, Mintz GS, Lerman A, et al. Tissue characterisation using intravascular radiofrequency data analysis: recommendations for acquisition, analysis, interpretation and reporting. Eurolntervention 2009;5:177-89. 
17. Hayashi $T$, Kiyoshima $T$, Matsuura $M$, et al. Plaque erosion in the culprit lesion is prone to develop a smaller myocardial infarction size compared with plaque rupture. Am Heart J 2005;149:284-90.

18. Hong MK, Mintz GS, Lee CW, et al. The site of plaque rupture in native coronary arteries: a three-vessel intravascular ultrasound analysis. J Am Coll Cardio/ 2005;46:261-5.

19. Fujii K, Masutani M, Okumura $T$, et al. Frequency and predictor of coronary thin-cap fibroatheroma in patients with acute myocardial infarction and stable angina pectoris a 3-vessel optical coherence tomography study. J Am Coll Cardio/ 2008;52:787-8.

20. Kume $T$, Okura $H_{1}$ Yamada $R$, et al. Frequency and spatial distribution of thin-cap fibroatheroma assessed by 3 -vessel intravascular ultrasound and optical coherence tomography: an ex vivo validation and an initial in vivo feasibility study. Circ J 2009;73:1086-91.
21. Toutouzas K, Karanasos A, Riga M, et al. Optical coherence tomography assessment of the spatial distribution of culprit ruptured plaques and thin-cap fibroatheromas in acute coronary syndrome. Eurolntervention 2012;8:477-85.

22. Schoenhagen $P$, Ziada KM, Kapadia SR, Crowe TD, Nissen SE, Tuzcu EM. Extent and direction of arterial remodeling in stable versus unstable coronary syndromes : an intravascular ultrasound study. Circulation 2000;101:598-603.

23. Inaba S, Mintz GS, Farhat NZ, et al. Impact of positive and negative lesion site remodeling on clinical outcomes: insights from PROSPECT. JACC Cardiovasc Imaging 2014;7:70-8.

24. Kramer MC, Rittersma SZ, de Winter RJ, et al. Relationship of thrombus healing to underlying plaque morphology in sudden coronary death. J Am Coll Cardiol 2010;55:122-32. 